\title{
Endoluminal vessel reconstruction by stent placement in patients with obstructive coronary artery disease who are poor surgical candidates
}

\author{
E Eeckhout, J-C Stauffer, P Vogt, L Kappenberger, J-J Goy
}

\begin{abstract}
Objective-To investigate the safety and efficacy of endoluminal vessel reconstruction by intracoronary stenting in patients with single or multivessel disease who have been turned down for surgical revascularisation.
\end{abstract}

Design-An observational, single centre trial of consecutive patients being followed up clinically.

Setting-Tertiary referral centre.

Patients-43 patients treated between 1995 and 1996 with a clinical follow up period of at least six months.

Main outcome measures-Acute procedural success following intervention (defined as a residual stenosis of $<30 \%$ with normal antegrade flow and without acute clinical complications); major adverse clinical events (death, myocardial infarction, repeat intervention, clinical restenosis); and functional status during follow up.

Results-Acute procedural success was achieved in 39 patients. Failures resulted from a refractory no reflow phenomenon with $Q$ wave myocardial infarction (two patients), and from subacute stent thrombosis (one patient) and saphenous vein graft rupture at stent site (one patient), both manifesting by non- $Q$ wave myocardial infarction. During a follow up period of 17 (8) months (mean (SD)), three of these patients died. Clinical restenosis was treated by repeat percutaneous intervention in 11 patients, while 28 patients remained asymptomatic. On a KaplanMeier estimate, the proportion of patients without adverse clinical events at two years was $65 \%$.

Conclusions-Percutaneous reconstruction of diseased coronary arteries in patients with single or multivessel disease turned down for surgical revascularisation may be considered as a valuable treatment option. On follow up, more events seem to be encountered than after elective de novo stenting of discrete coronary lesions. This is probably related to higher clinical restenosis rates. (Heart 1998;80:355-358)

Keywords: coronary artery disease; stents; restenosis

Catheter based therapeutic interventions were first applied in patients with coronary artery disease 20 years ago. ${ }^{1}$ A decade after the intro- duction of balloon angioplasty, intracoronary placement of metallic stents was proposed as an alternative to repeat angioplasty or coronary artery bypass grafting for the treatment of restenosis in native coronary arteries and in saphenous vein grafts. ${ }^{2}$ It soon became apparent that the mechanical properties of this device enabled the acute restoration of adequate antegrade flow in abrupt or threatened vessel closure during angioplasty. ${ }^{2}$ Another decade later, this technique has been recognised as an essential breakthrough in interventional cardiology. Randomised trials on primary restenosis prevention repeatedly showed lower restenosis rates following elective Palmaz-Schatz placement than after conventional angioplasty. ${ }^{3}$ Moreover, with more adequate postprocedural pharmacological regimens and better stent deployment, this technique now has an excellent short term clinical outcome which may even surpass the results obtained with angioplasty.

Because of this, the indications for stenting have been widened to include patients for percutaneous revascularisation who would formerly only have been treated medically. The scaffolding properties of stents, which are now customised in size and length, allow coronary artery reconstruction. In this report we describe our initial experience of the use of stents in patients who were considered poor candidates for surgical revascularisation.

\section{Methods}

PATIENT POPULATION

Patients with symptomatic, documented ischaemia and single or multivessel coronary artery disease who were turned down for coronary artery bypass grafting were considered for vessel reconstruction. Decisions not to operate were taken by consensus between surgeons and cardiologists during staff meetings. Eligible patients were symptomatic despite optimal medical treatment or else because concomitant disease precluded the use of specific antianginal drugs. Ischaemia and myocardial viability were demonstrated by conventional stress testing, stress echocardiography, and myocardial scintigraphy. From a technical surgical point of view, the angiographic appearance of the coronary arteries-long lesions, diffuse disease, poor or invisible distal bed because of absent collateralisation-was the main reason for deciding not to operate, but additional concomitant disease could be a further element in the final decision. Patients were not eligible for percutaneous intervention if 
Table 1 Clinical and angiographic characteristics of the study patients

\begin{tabular}{lc}
\hline Characteristic & $n$ \\
\hline Coronary risk factors and concomitant disease & \\
Hypercholesterolaemia & 25 \\
Hypertension & 21 \\
Diabetes mellitus & 11 \\
Family history & 19 \\
Smoking & 14 \\
Previous myocardial infarction & 14 \\
Previous bypass grafting & 15 \\
Angina class (CCS classification) & \\
II & 2 \\
III & 15 \\
IV & 26 \\
Antianginal medication & 21 \\
Two drugs & 22 \\
Three drugs & $0.51(0.21)$ \\
Left ventricular ejection fraction (mean (SD)) & \\
Number of vessels diseased & 9 \\
One & 17 \\
Two & 17 \\
Three & 15 \\
Vessel type & 6 \\
Left anterior descending & 19 \\
Left circumflex & 14 \\
Right coronary artery & \\
Saphenous vein graft & \\
CCS, Canadian Cardiovascular Society. &
\end{tabular}

antiplatelet agents or short term heparin infusion could not be prescribed; or the vessel reference diameter was estimated to be less than $2 \mathrm{~mm}$.

INTERVENTION AND FOLLOW UP

We attempted to identify the "culprit" lesion in patients with multivessel involvement by noninvasive means (ECG changes during anginal pain, stress echocardiography). Otherwise, staged or single session procedures were undertaken in these patients. Various stents were available to the operators: the PalmazSchatz stent (Johnson and Johnson Interventional, Warren, New Jersey, USA), the Wallstent (Schneider AG, Bülach, Switzerland), the Multilink stent (ACS, Santa Clara, California, USA), the SCIMED NIR stent (Boston Scientific, Galway, Ireland), the Be stent and Wiktor stent (Medtronic, Lausanne, Switzerland), and the Micro stent (AVE, Santa Rosa, California, USA). They were used at the discretion of the operator and chosen according to adequate size and length. Technically, attempts were made to cover both the proximal and the distal ends of

Table 2 Technical details related to the intervention

\begin{tabular}{lc}
\hline Characteristic & $n$ \\
\hline Number of vessels stented & \\
Single & 32 \\
Two & 11 \\
Number of stents/vessel & 11 \\
Single & 17 \\
Two & 19 \\
Three & 6 \\
Four & 1 \\
More than four & 132 \\
Total & \\
Stent combinations & 16 \\
Microstent only & 14 \\
Palmaz-Schatz/Microstent & 4 \\
Wallstent only & 7 \\
Wallstent/Microstent & 6 \\
NIR/Microstent & 2 \\
Be stent only & 3 \\
Multilink/Microstent & 3 \\
Wiktor/Microstent & 1 \\
Wiktor/Wallstent & 1 \\
Palmaz-Schatz/Wallstent and Microstent & \\
\hline
\end{tabular}

the lesions by using long single or multiple serial stents. These were implanted according to the manufacturer's recommendations, attempting adequate stent expansion by additional high pressure, non-compliant balloon angioplasty within the stent (if required) and guided by on-line quantitative angiographic analysis aiming at $<30 \%$ residual stenosis. Intravascular ultrasound was not performed.

Following the procedure, no additional heparin was given, and the arterial sheath was removed as soon as the activated clotting time was longer than 150 seconds. Patients were discharged on ticlopidine (500 mg daily for four weeks) and aspirin $100 \mathrm{mg}$ daily. They were followed up clinically by their referring cardiologists on a regular basis. Repeat angiography at six months was not performed systematically.

MAIN OUTCOME MEASURES

Acute procedural success was defined as the absence of clinical complications (death, myocardial infarction, reintervention) combined with angiographic success (defined as a residual stenosis of $<30 \%$ with normal antegrade flow).

Major adverse clinical events (death, myocardial infarction, repeat intervention, clinical restenosis) were recorded during the entire follow up period.

Angina functional status (according to the Canadian Cardiovascular Society classification) was recorded during the follow up.

\section{STATISTICAL ANALYSIS}

Continuous variables are expressed as means (SD). The proportion of patients without adverse events during follow up were estimated by the Kaplan-Meier method.

\section{Results}

PATIENT POPULATION

This study was conducted between January 1995 and December 1996, providing a follow up of at least six months for each patient. The study population consisted of 43 patients (38 male, five female) with a mean (SD) age of 69 (15) years, out of a total cohort of 611 patients (7\%) treated by endoluminal stenting during the study period. Baseline clinical and angiographic characteristics of the study population are given in table 1 . The majority of patients presented with severe angina and multivessel involvement. According the American College of Cardiology/American Heart Association classification all stenoses were judged to be type C lesions.

Diffuse coronary artery disease with long and multiple lesions and a poor distal vascular bed was always present (and was the only factor in 10 patients). Additional reasons for refusing surgical intervention were: postoperative graft closure (two patients), morbid obesity (body mass index $>35 \mathrm{~kg} / \mathrm{mm}^{2}$, three patients), advanced age ( $>75$ years, nine patients), repeated coronary artery bypass grafting (four patients), poor left ventricular function (four patients), severe chronic obstructive lung 

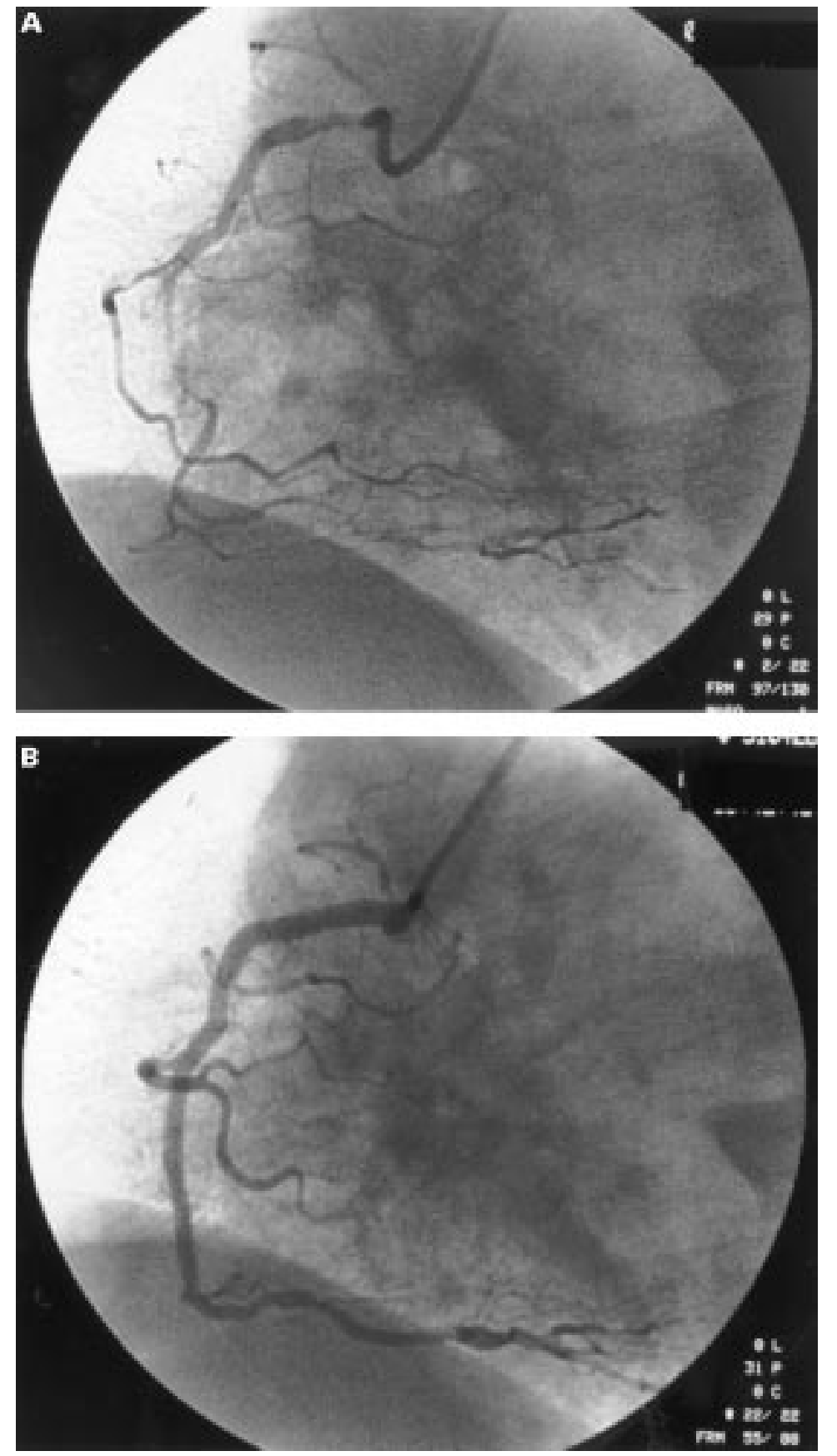

Figure 1 Vessel reconstruction of the right coronary artery. (A) Diffuse disease of the right coronary artery with lesions at proximal and mid sites, and distal subocclusion; $(B)$ vessel reconstruction by stent placement proximal and mid sites.

disease (three patients), haematological disorder (one patient), refractory unstable angina (three patients), and neoplasms (four patients).

INTERVENTION

Technical details related to the intervention are shown in table 2. Multivessel stenting was attempted in $26 \%$ of the cases. Vessel reconstruction required multiple stent placement in most of the patients, with a mean of 2.5 stents per vessel treated. Although the procedure was guided by quantitative angiographic analysis, classic quantitative variables such as reference diameter or minimum luminal diameter cannot be provided because of the particular anatomy of the study population.

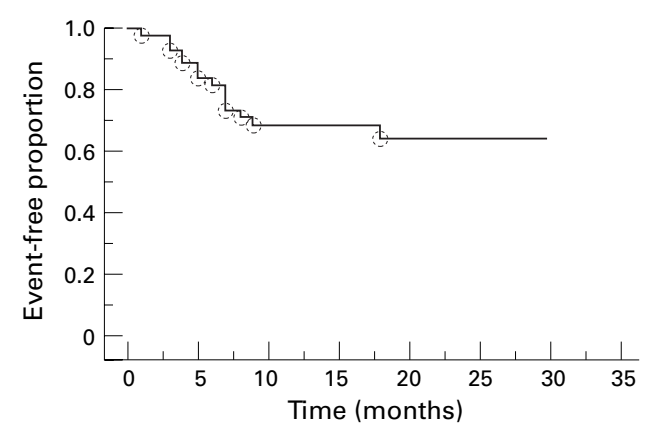

Figure 2 Cumulative distribution curve of the cardiac event free proportion extended at 30 months' follow up.

Complications occurred in four patients: a refractory no reflow phenomenon leading to $\mathrm{Q}$ wave myocardial infarction in two patients with saphenous vein grafts lesions, subacute stent thrombosis requiring urgent reintervention and leading to a non-Q wave myocardial infarction in one patient, and saphenous vein graft rupture after high pressure angioplasty for stent expansion in one patient.

A typical example of vessel reconstruction in the right coronary artery is shown in fig 1 .

FOLLOW UP

During a mean (SD) follow up of 17 (8) months, three patients died. Two died suddenly at three weeks and four months and their deaths were considered to be procedure related. One patient died of heart failure at 18 months. Because of angina recurrence, repeat angiography was performed in 15 patients and angiographic restenosis was detected in 11 . Restenosis was classified as focal (lesion length $\leqslant 15 \mathrm{~mm}$ ) (five patients) versus diffuse (lesion length $>15 \mathrm{~mm}$ ) (six patients). Repeat percutaneous intervention was performed in these patients by means of conventional angioplasty (10 patients) and stenting within the stent (one patient). A second reintervention was required in two patients. In one of these, the indication for surgical intervention was reconsidered and accepted. Vascular reconstruction had allowed a better evaluation of the distal vascular bed and bypass grafting was considered technically feasible.

MAIN OUTCOME MEASURES

Procedural success was obtained in 39 patients; early complications occurred in four. During follow up, major adverse event occurred in 14 patients: three patients died, and repeat intervention was performed in 11 . According to the Canadian Cardiovascular Society classification, 28 patients were asymptomatic during follow up. The proportion of patients without clinical events was estimated to be $65 \%$ at two years (fig 2).

\section{Discussion}

In this study we show the feasibility and efficacy of endoluminal reconstruction of severely diseased coronary arteries in patients considered to be poor candidates for surgical revascularisation, with a low incidence of acute complications and an acceptable clinical restenosis rate. 
From 1995 on, the acute clinical outcome of patients treated by emergency or elective coronary stent placement has improved dramatically because of more nearly optimal stent expansion and because of more adequate antiplatelet treatment after intervention. ${ }^{4}$ Of even greater importance, tremendous progress has been made in stent delivery systems, making them flexible, extremely trackable, easier to use on rapid exchange catheters, and customised in size and length. The indications for stenting have therefore increased as cardiologists attempt percutaneous intervention in patients previously given medical treatment only.

Patients with long and diffuse coronary artery lesions may theoretically benefit from stent placement, as the mechanical properties of the device permit reconstruction of the diseased segments. A previous study (published in abstract form only) focused on this, though it mainly reported acute results: Karam et al found a $92.1 \%$ procedural success rate in 101 patients treated by multiple overlapping Gianturco-Roubin stents, which is comparable with the $91 \%$ success rate reported here. ${ }^{5}$

At follow up, Kornowski and colleagues reported a $16.4 \%$ target site revascularisation rate in patients receiving at least three stents in the same vessel, while we observed a $27.5 \%$ clinical restenosis rate. ${ }^{6}$ Among 41 patients treated by radical endovascular reconstruction by stenting, Savage et al showed that $70 \%$ of patients were asymptomatic at one year. ${ }^{7}$ In the present study, $70 \%$ of patients were free from symptoms at six months. The need for target site revascularisation after using multiple stents seems greater than after elective single stent placement and the proportion of asymptomatic patients lower. This seems inherent to the procedure, which implies that more aggressive dilatation causes a higher degree of vascular trauma and subsequent neointimal proliferation.

In the present series, complications occurred mainly in patients with saphenous vein graft disease. Refractory no-reflow was observed in two patients despite intracoronary administration of nitrates, verapamil, and thrombolysis. Administration of abciximab before interven- tion, which has emerged as an efficient additional treatment in patients with high risk angioplasty, might have been useful in these patients. ${ }^{8}$ This option was, however, not a standard treatment at the time of the procedure. Kelly et al performed vascular reconstruction in 26 patients with saphenous vein graft disease and observed reduced TIMI flow in two, one of whom had a myocardial infarct. ${ }^{9}$

Several limitations related to this study need to mentioned. First, the study population is rather small. Second, a selection bias was certainly present as the criteria for surgical refusal were centre and operator dependent. Finally, intravascular ultrasound guidance might have been useful in certain cases for appropriate stent size selection (to avoid vessel rupture) and to ensure adequate stent expansion.

In conclusion, endovascular reconstruction for patients with diffuse or severe coronary artery disease considered not suitable for surgical revascularisation may be attempted with a high degree of acute success and an acceptable need for late target site revascularisation.

1 Gruentzig AR, Senning A, Siegenthaler W. Nonoperative dilatation of coronary artery stenosis. $N$ Engl $\mathcal{f}$ Med 1979;301:61-8

2 Sigwart U, Puel J, Mirkovitch V, et al. Intravascular stents to prevent occlusion and restenosis after transluminal angioplasty. N Engl F Med 1987;316:701-6.

3 de Jaegere PPT, Eefting FD, Popma JJ, et al. Clinical trials on intracoronary stenting. Semin Intervent Cardiol 1996;1: 233-45.

4 Mak KH, Belli G, Ellis SG, et al. Subacute stent thrombosis: evolving issues and current concepts. $7 \mathrm{Am}$ Coll Cardiol 1996;27:494-503.

5 Karam C, Loubeyre C, Cassagneau B, et al. Is endoluminal coronary reconstruction using overlapped GianturcoRoubin stents to treat long complex lesions an acceptable procedure? [abstract.] Eur Heart f 1996;17(suppl): 218

6 Kornowski R, Mintz GS, Mehran R, et al. The "full metal jacket": procedural results and late clinical outcomes after placement of three or more coronary stents [abstract]. $\mathcal{F}$ Am Coll Cardiol 1997;suppl I:788.

7 Savage M, Fernandes L, Fischman D, et al. Radical endoluminal reconstruction of diffusely diseased coronary arteries using multiple stents [abstract]. Circulation 1996;94(suppl I): 258 .

8 The EPILOG investigators. Platelet glycoprotein IIb/IIIa receptor blockade and low-dose heparin during percutaneous coronary revascularization. $N$ Engl f Med 1997;336: 1689-96.

9 Kelly PA, Kurbaan As, Clague JR, et al. Total endovascular reconstruction of occluded saphenous vein grafts using coronary of peripheral Wallstents. F Invas Cardiol 1997;9: $513-17$ 